\title{
Espessura Íntima-Média Carotídea como Marcador de Risco Cardiovascular em Pacientes Com Hipotireoidismo Subclínico
}

\section{perspectivas}

\author{
Carla A. de Almeida \\ Patrícia de F. dos S. TeIXeIRA \\ DÉbORA V. SOARES \\ MÔNICA D. Cabral \\ SHELLA M. DA COSTA \\ ELIZABETH F. DE SALLES \\ Nathalie A. de O. e Silva \\ FELIPE F.C. DE MORAIS \\ AleXANDru BUescu \\ Jodélia M. HenRiQues \\ MARIO VAISMAN
}

Serviço de Endocrinologia,

Faculdade de Medicina do

Hospital Universitário Clementino

Fraga Filho, Universidade Feder-

al do Rio de Janeiro, RJ.

Recebido em 03/05/06

Revisado em 18/09/06

Aceito em 22/11/06

\section{RESUMO}

O hipotireoidismo subclínico (HS) já foi associado a aumento do risco cardiovascular. $\mathrm{Na}$ avaliação desse risco, a medida da espessura íntima-média (EIM) carotídea por ultra-sonografia é capaz de detectar alterações iniciais da aterosclerose. $\mathrm{O}$ objetivo deste estudo foi avaliar a EIM carotídea em pacientes com HS e sua associação com um provável aumento do risco cardiovascular. Não foi encontrada diferença significativa nas medidas da EIM das pacientes com HS e das controles. Os resultados encontrados nos dois grupos estudados foram, respectivamente: $0,573 \pm 0,070 \mathrm{~mm}$ vs. $0,576 \pm 0,068 \mathrm{~mm}$ para as carótidas comuns $(p=0,904)$ e $0,602 \pm 0,079 \mathrm{~mm}$ vs. $0,617 \pm 0,102 \mathrm{~mm}$ para as bifurcações $(p=0,714)$. Mesmo após estratificação das pacientes de acordo com o TSH e com a presença ou não de auto-imunidade, a diferença entre os sub-grupos permaneceu sem significância estatística. As medidas da EIM nesses grupos nos sítios avaliados foram: TSH 4-8 $\mathrm{mUl} / \mathrm{L}: 0,579 \pm 0,070 \mathrm{~mm}$ e 0,586 \pm $0,063 \mathrm{~mm}$; TSH > $8 \mathrm{mUI} / \mathrm{L}: 0,569 \pm 0,073 \mathrm{~mm}$ e 0,616 $\pm 0,091 \mathrm{~mm}$; anti-TPO+:

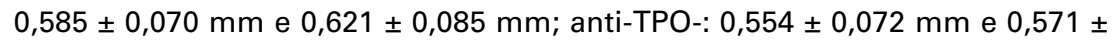
$0,066 \mathrm{~mm}$. Também não houve diferença no lipidograma e nas dosagens de apoproteína B e de lipoproteína (a). Este fato sugere que o HS, quando leve, sem alterações metabólicas associadas, não promove aumento do risco cardiovascular. (Arq Bras Endocrinol Metab 2007;51/3:472-477)

Descritores: Hipotireoidismo; Artérias carótidas; Lipídeos; Cardiovascular

\section{ABSTRACT}

Carotid Intima-Media Thickness as a Marker of Cardiovascular Risk in Patients With Subclinical Hypothyroidism.

Subclinical hypothyroidism (SH) has been associated with an increased risk for coronary disease. Carotid intima-media thickness (IMT), as assessed by ultrasonography, is a precise marker of atherosclerotic changes and can be used as an endpoint for cardiovascular events. Aims of this study were to determine carotid IMT in a group of patients with $\mathrm{SH}$ and its possible association with an increase in cardiovascular risk. There were no significant differences in mean carotid IMT between patients and controls. Results of both groups were, respectively: common carotid arteries, $0.573 \pm 0.070 \mathrm{~mm}$ and $0.576 \pm 0.068 \mathrm{~mm}$ $(p=0.904)$; carotid bifurcation, $0.602 \pm 0.079 \mathrm{~mm}$ and $0.617 \pm 0.102 \mathrm{~mm}(\mathrm{p}=$ $0.714)$. Similar results were obtained when analyzing subgroups with serum $\mathrm{TSH}<$ or $>8 \mathrm{mIU} / \mathrm{L}$ and with positive or negative titers of TPOAb. The mean carotid IMT in these subgroups were: TSH $4-8 \mathrm{mIU} / \mathrm{L}: 0.579 \pm 0.070 \mathrm{~mm}$ and $0.586 \pm 0.063 \mathrm{~mm} ; \mathrm{TSH}>8 \mathrm{mlU} / \mathrm{L}: 0.569 \pm 0.073 \mathrm{~mm}$ and $0.616 \pm 0.091 \mathrm{~mm}$; TPOAb+: $0.585 \pm 0.070 \mathrm{~mm}$ and $0.621 \pm 0.085 \mathrm{~mm}$; TPOAb-: $0.554 \pm 0.072 \mathrm{~mm}$ and $0.571 \pm 0.066 \mathrm{~mm}$. No differences in the lipid profile and in the apoprotein $B$ and lipoprotein (a) levels between the groups were found. These findings suggest that mild $\mathrm{SH}$ with no related metabolic changes is not associated with an increase in cardiovascular risk, as assessed by carotid IMT. (Arq Bras Endocrinol Metab 2007;51/3:472-477)

Keywords: Hypothyroidism; Carotid arteries; Lipids; Cardiovascular 
$\mathrm{H}$ IPOTIREOIDISMO SUBCLÍNICO (HS) é definido como a presença de níveis elevados de tireotrofina (TSH) associada a níveis de tiroxina livre (T4l) dentro dos limites da normalidade (1). Acomete $1 \%$ a $10 \%$ da população adulta, de acordo com vários estudos de prevalência (2-5), podendo atingir uma prevalência maior em mulheres idosas (5).

Como os pacientes que apresentam HS são oligo ou assintomáticos, sua relevância clínica ainda é controversa. Entretanto, existe possível associação com aumento dos níveis séricos de colesterol total (CT), da lipoproteína de baixa densidade (LDL) (6-8) e do risco para doença cardiovascular e arterial periférica (2). Existe, também, a possibilidade de ocorrer progressão para hipotireoidismo manifesto, sabidamente associado à aterosclerose e aumento do risco cardiovascular (9), entre outras alterações.

A hipercolesterolemia, principalmente o aumento dos níveis de LDL, é descrita como o fator de risco mais importante para o desenvolvimento da aterosclerose (10). Porém, atualmente, outros parâmetros laboratoriais, como a apoproteína $\mathrm{B}$ (apoB) (11-13) e a lipoproteína (a) (Lpa) $(14,15)$, têm sido apontados como marcadores importantes na avaliação deste risco.

Inicialmente, a aterosclerose progride na parede arterial promovendo seu espessamento, sem redução do diâmetro luminal (16). Algumas técnicas não-invasivas vêm sendo utilizadas na avaliação de todas as suas fases, inclusive as mais precoces. Dentre elas, a quantificação da espessura da íntima-média (EIM) carotídea utilizando imagens ultra-sonográficas é um método seguro, sem exposição à radiação e de baixo custo, que permite uma avaliação direta de todas as fases da aterosclerose, sendo útil tanto no seu diagnóstico como no seu acompanhamento (17-19).

A medida da EIM carotídea pode ser utilizada na pesquisa de doença cardiovascular subclínica, mesmo na ausência de outros fatores de risco (20-22), sendo, atualmente, o único método recomendado pela Ameri can Heart Association para avaliação de risco cardiovascular (23). De fato, já foi demonstrado que para cada $0,1 \mathrm{~mm}$ de aumento da EIM há um incremento de $11 \%$ (IC 95\%, 6\% a $16 \%$ com $\mathrm{p}<0,001$ ) no risco de infarto agudo do miocárdio (IAM) (24) e que um incremento na EIM de 0,03 $\mathrm{mm}$ ao ano está relacionado a um risco relativo de 3,1 para a ocorrência de morte por doença coronariana, IAM não-fatal e revascularização miocárdica (25). Além disso, alguns autores acreditam que valores inferiores a $0,8 \mathrm{~mm}$ não estariam associados a complicações cardiovasculares (26).

Apesar de ser examinador dependente, o uso da ultra-sonografia para quantificação da EIM carotídea possui boa reprodutibilidade, conforme já evidenciado por Bots e cols. (27) e por Kanters e cols. (28).

O objetivo deste estudo foi avaliar a presença de alterações precoces de aterosclerose por meio da quantificação da EIM das carótidas por ultra-sonografia em pacientes com HS. Foram também avaliados outros parâmetros que poderiam interferir na medida da EIM e contribuir para o aumento do risco cardiovascular, como o perfil lipídico, a apoproteína B (apoB) e a lipoproteína (a) (Lpa). Os resultados foram comparados com os encontrados em indivíduos sem disfunção tireoideana e com mesmo sexo, idade e índice de massa corporal (IMC).

\section{PACIENTES E MÉTODOS}

\section{Pacientes}

Foram avaliadas 57 mulheres, sendo 30 com HS e 27 eutireoidianas, sem história de doença tireoidiana e com auto-imunidade negativa. A etiologia do HS foi tireoidite auto-imune em 17 pacientes e indeterminada nas outras 13 , visto que a pesquisa de auto-anticorpos foi negativa em 12 pacientes, não tendo sido realizada em uma paciente. Não havia história de tratamento prévio para disfunção tireoidiana em nenhuma paciente. A média de idade das pacientes foi $43,07 \pm 9,76$ anos, variando de 19 a 59 anos, e das controles, $43,19 \pm 8,39$ anos $(\mathrm{p}=0,961)$, variando de 28 a 57 anos; a mediana foi 44 anos nos dois grupos. O IMC foi $27,32 \pm 4,61 \mathrm{~kg} / \mathrm{m}^{2}$ e $25,4 \mathrm{l} \pm 4,38 \mathrm{~kg} / \mathrm{m}^{2}(\mathrm{p}=0,117)$, respectivamente.

O diagnóstico do HS foi feito após duas dosagens de TSH e T4l, com no mínimo seis semanas de intervalo entre elas. Como critério de exclusão, utilizamos uso de levotiroxina, doença cardiovascular, hipertensão arterial sistêmica (HAS), diabetes mellitus e tabagismo. Todos assinaram o termo de consentimento livre e esclarecido aprovado pelo Comitê de Ética e Pesquisa do Hospital Universitário Clementino Fraga Filho (HUCFF).

Para análise da influência do nível de TSH e da presença de auto-imunidade tireoidiana na EIM carotídea, as pacientes foram estratificadas de acordo com os níveis de TSH em dois grupos (TSH entre 4,0 e 8,0 mUI/L e acima de $8 \mathrm{mUI} / \mathrm{L}$ ) e em outros dois grupos, com positividade para anticorpos anti-peroxidade (anti-TPO) ou não.

\section{Métodos}

O TSH foi dosado por ensaio imunométrico (IMMULITE $\left.\mathrm{DPC}^{\circledR}\right)$, com valor de referência (VR) de 0,4 a $4,0 \mathrm{mUI} / \mathrm{L}$, sensibilidade de $0,002 \mathrm{mUI} / \mathrm{L}$, coeficiente de variação $(\mathrm{CV})$ inter-ensaio de $12,5 \%$ a $6,4 \%$ e intra-ensaio, de $2,5 \%$ a $5,1 \%$, para valores entre 0,016 a $39 \mathrm{mUI} / \mathrm{L}$. A dosagem de T4l foi feita por quimioluminescência (IMMULITE DPC ${ }^{\circledR}$ ), com VR de 0,8 a 1,9 ng/dL, sensibilidade de 0,15 ng/dL, CV inter-ensaio de $9,0 \%$ a $7,7 \%$ e intra-ensaio, de $7,5 \%$ a $5,2 \%$, 
para valores entre 0,67 e 5,2 ng/dL. Para avaliação de autoimunidade foi dosado o anticorpo anti-TPO, por ensaio imunométrico por quimioluminescência (IMMULITE DPC $\left.2000{ }^{\circledR}\right)$. O CV intra-ensaio foi de $4,3 \%$ a $5,6 \%$ e o interensaio, de $7,8 \%$ a $10,5 \%$. São considerados normais valores inferiores a $35 \mathrm{UI} / \mathrm{mL}$.

A dosagem de colesterol total (CT), da lipoproteína de alta densidade (HDL) e dos triglicerídeos (TG) foi realizada por ensaio colorimétrico enzimático, Roche/Hitachi ${ }^{\circledR}$. Para o CT, o intervalo de medição foi de 3 a $800 \mathrm{mg} / \mathrm{dL}$, a variação intra-ensaio, de $0,8 \%$ e a inter-ensaio, de $1,7 \%$. Para o HDL, esses valores foram de 3 a $120 \mathrm{mg} / \mathrm{dL}, 0,90 \%$ e $1,85 \%$ e para os TG, 4 a $1000 \mathrm{mg} / \mathrm{dL}, 1,5 \%$ e $1,8 \%$, respectivamente. Os níveis de LDL foram calculados através da fórmula de Friedewald (29), da seguinte forma: $\mathrm{LDL}=\mathrm{CT}-(\mathrm{HDL}+\mathrm{TG} / 5)$.

A apoproteína $\mathrm{B}$ foi dosada por nefelometria, com kit Dade Behring, $\mathrm{BN}^{\circledR}$, com VR de 30 a $100 \mathrm{mg} / \mathrm{dL}, \mathrm{CV}$ intra-ensaio de $1,9 \%$ e inter-ensaio, de $2,4 \%$.

A dosagem de Lpa também foi realizada por nefelometria, com Kit Dade Behring, $\mathrm{BN}^{\circledR}$, sendo considerados normais valores até $30 \mathrm{mg} / \mathrm{dL}$. Os $\mathrm{CV}$ intra-ensaio e inter-ensaio foram de $4,1 \%$ e $3,1 \%$.

A ultra-sonografia das carótidas para avaliação da EIM foi realizada utilizando-se um aparelho eco-color Doppler de alta resolução (Acusson, Modelo Aspem Advanced) com transdutor linear multifreqüência de $10 \mathrm{MHz}$. A imagem característica observada é de duas linhas paralelas ecogênicas separadas por um espaço relativamente hipoecogênico. A EIM foi definida como a distância entre a borda principal da primeira linha ecogênica e a borda principal da segunda linha ecogênica, como descrito por Pignoli e cols. (30). As medidas foram efetuadas nas carótidas comuns e bifurcações, tendo sido utilizada para análise a média dos valores encontrados nos lados direito e esquerdo de cada sítio. Todos os exames foram realizados pelo mesmo observador, que desconhecia os dados clínicos dos indivíduos avaliados, com CV [desvio-padrão (DP)/média] intra-operador de $7,7 \pm 4,3 \%$.

\section{Análise estatística}

Os dados referentes às variáveis contínuas foram expressos como média \pm desvio-padrão, exceto quando indicado. Foi utilizado o programa SPSS, versão 10, na análise estatística. As comparações das médias entre os dois grupos de estudo foram feitas utilizando-se o teste $\mathrm{t}$ de Student para as variáveis com distribuição sabidamente normal na população (idade, IMC, CT, HDL, TG e LDL). O teste de MannWhitney foi utilizado para comparação das médias de EIM, TSH, T4L, apoB e Lpa entre os dois grupos. O grupo de pacientes com HS foi subdividido entre os com TSH entre 4,0 e 8,0 mUI/L e os com TSH acima de 8,0 mUI/L, para avaliação de possível influência do nível do TSH nos resultados. Os pacientes também foram divididos em outros dois sub-grupos, de acordo com a presença ou não de anticorpos anti-TPO. Na análise das médias entre os três sub-grupos, foi utilizada ANOVA para variáveis sabidamente de distribuição normal ou Kruskal-Wallis. O nível de significância estatístico aceito foi de $5 \%$.

\section{RESULTADOS}

O TSH médio das pacientes foi $8,69 \pm 3,82 \mathrm{mUI} / \mathrm{L}$ (variando de 4,3 a 20,8 mUI/L) e das controles, 1,53 \pm $0,75 \mathrm{mUI} / \mathrm{L}(\mathrm{p}<0,001)$, enquanto que o T4l foi $1,03 \pm$ $0,19 \mathrm{ng} / \mathrm{dL}$ e $1,23 \pm 0,20 \mathrm{ng} / \mathrm{dL}$, respectivamente $(\mathrm{p}<$ $0,001)$. Das 30 pacientes, 14 apresentavam TSH entre 4 e $8 \mathrm{mUI} / \mathrm{L}$ e 16, TSH acima de $8 \mathrm{mUI} / \mathrm{L}$ (em 7, o TSH se encontrava acima de $10 \mathrm{mUI} / \mathrm{L}) ; 17$ (56,7\%) apresentavam positividade para o anti-TPO, 12 (40,0\%), dosagem deste anticorpo dentro dos limites da normalidade e em $1(3,3 \%)$ essa pesquisa não foi realizada.

No grupo com HS e nos controles, as médias de colesterol total foram, respectivamente, 213,00 \pm $51,85 \mathrm{mg} / \mathrm{dL}$ e $200,74 \pm 33,30 \mathrm{mg} / \mathrm{dL}(\mathrm{p}=0,289)$, as de HDL, 55,20 $\pm 10,59$ e $52,96 \pm 11,92(\mathrm{p}=$ $0,456)$, as de TG, $119,07 \pm 68,54 \mathrm{mg} / \mathrm{dL}$ e $106,11 \pm$ $49,44 \mathrm{mg} / \mathrm{dL}(\mathrm{p}=0,421)$, as de LDL, 133,73 $\pm 46,60$ $\mathrm{mg} / \mathrm{dL}$ e $126,30 \pm 32,07 \mathrm{mg} / \mathrm{dL}(\mathrm{p}=0,482)$, as de apoB, $116,97 \pm 39,78 \mathrm{mg} / \mathrm{dL}$ e $106,07 \pm 26,06$ $\mathrm{mg} / \mathrm{dL}(\mathrm{p}=0,37 \mathrm{l})$ e as de Lpa, 42,31 $\pm 48,54 \mathrm{mg} / \mathrm{dL}$ e $25,38 \pm 19,21 \mathrm{mg} / \mathrm{dL}(\mathrm{p}=0,462)$.

As características clínicas e laboratoriais das participantes encontram-se na tabela 1 .

A média dos valores de EIM das pacientes obtidos nas carótidas comuns foi $0,573 \pm 0,070 \mathrm{~mm}$ e, nas bifurcações, 0,602 $\pm 0,079 \mathrm{~mm}$. Nas controles, os valores encontrados foram $0,576 \pm 0,068 \mathrm{~mm}$ e 0,617 $\pm 0,102$ $\mathrm{mm}$, respectivamente. Não houve diferença estatística entre os grupos $(\mathrm{p}=0,904$ e $\mathrm{p}=0,714$, para os dois sítios avaliados). Em uma paciente, foi observada a presença de placa fibrolipídica promovendo obstrução inferior a 30\% do fluxo sanguíneo na bifurcação esquerda. Nesta paciente, apenas a EIM carotídea do lado direito foi utilizada no cálculo da média da EIM nas bifurcações. Nenhuma controle apresentou placa em qualquer sítio avaliado.

Mesmo após estratificação das pacientes com HS em dois grupos, de acordo com a faixa de TSH (entre 4 e $8 \mathrm{mUI} / \mathrm{L}$ e acima de $8 \mathrm{mUI} / \mathrm{L}$ ), as médias da EIM continuaram sem diferenças estatísticas entre os grupos e os controles. Os dados da ultra-sonografia estão descritos na tabela 2 .

Ao analisarmos as pacientes em dois grupos, com positividade para anti-TPO ou não, também não encontramos diferença estatística na EIM, resultados descritos na tabela 2 .

\section{DISCUSSÃO}

Este estudo evidenciou que pacientes do sexo feminino e de meia-idade com HS e sem história de outras condições que pudessem interferir no risco cardiovas- 
Tabela 1. Médias das variáveis clínicas e laboratoriais nos grupos com HS e controle (média \pm desvio-padrão).

\begin{tabular}{|c|c|c|}
\hline Variável & Pacientes $(n=30)$ & Controles $(n=27)$ \\
\hline Idade (anos)* & $43,07 \pm 9,76$ & $43,19 \pm 8,39$ \\
\hline $\operatorname{IMC}\left(\mathrm{kg} / \mathrm{m}^{2}\right)^{*}$ & $27,32 \pm 4,61$ & $25,41 \pm 4,38$ \\
\hline $\mathrm{TSH}(\mathrm{mUI} / \mathrm{L}) * *$ & $8,69 \pm 3,82$ & $1,53 \pm 0,75$ \\
\hline T4 livre $(\mathrm{ng} / \mathrm{dL})^{* *}$ & $1,03 \pm 0,19$ & $1,23 \pm 0,20$ \\
\hline Colesterol total $(\mathrm{mg} / \mathrm{dL})^{*}$ & $213,00 \pm 51,85$ & $200,74 \pm 33,30$ \\
\hline $\mathrm{HDL}(\mathrm{mg} / \mathrm{dL})^{*}$ & $55,20 \pm 10,59$ & $52,96 \pm 11,92$ \\
\hline $\operatorname{LDL}(\mathrm{mg} / \mathrm{dL})^{*}$ & $133,73 \pm 46,60$ & $126,30 \pm 32,07$ \\
\hline Triglicerídeos $(\mathrm{mg} / \mathrm{dL})^{*}$ & $119,07 \pm 68,54$ & $106,11 \pm 49,46$ \\
\hline Apoproteína B (mg/dL)* & $116,97 \pm 39,78$ & $106,07 \pm 26,06$ \\
\hline Lipoproteína a $(\mathrm{mg} / \mathrm{dL})^{*}$ & $42,31 \pm 48,53$ & $25,38 \pm 19,21$ \\
\hline
\end{tabular}

Tabela 2. Médias da EIM obtida nas carótidas comuns e bifurcações nos grupos com HS, divididos de acordo com a faixa do TSH e com a presença ou não de positividade para o anti-TPO e no grupo controle (média \pm desvio-padrão).

\begin{tabular}{lccc}
\hline & Grupo & \multicolumn{2}{c}{$\begin{array}{c}\text { Variável } \\
\text { EIM nas bifurcações } \\
\text { (mm) }\end{array}$} \\
& & $\begin{array}{c}\text { EIM nas carótidas } \\
\text { comuns }(\mathbf{m m})\end{array}$ & \\
Controle $(\mathrm{n}=27)$ & & $0,576 \pm 0,068$ & $0,617 \pm 0,102$ \\
TSH & Entre 4 e $8 \mathrm{mUl} / \mathrm{mL}(\mathrm{n}=14)$ & $0,579 \pm 0,070$ & $0,586 \pm 0,063$ \\
Anti-TPO & $>8 \mathrm{mUl} / \mathrm{mL}(\mathrm{n}=16)$ & $0,569 \pm 0,073$ & $0,616 \pm 0,091$ \\
& Positivo $(n=17)$ & $0,585 \pm 0,070$ & $0,621 \pm 0,085$ \\
& Negativo $(n=12)$ & $0,554 \pm 0,072$ & $0,571 \pm 0,066$ \\
\hline
\end{tabular}

Quando se comparou EIM nas carótidas comuns e nas bifurcações dos grupos divididos de acordo com a faixa de TSH com o grupo controle, obteve-se $p=0,904$ e $p=0,649$, respectivamente.

Quando se comparou EIM nas carótidas comuns e nas bifurcações dos grupos com positividade ou não para anti-TPO com o grupo controle, obteve-se $p=0,283$ e $p=0,166$, respectivamente.

cular, não apresentavam a medida da EIM mais elevada do que as controles. Além disso, não foi encontrada diferença estatisticamente significante no perfil lipídico e nos níveis de apoB e Lpa. Após estratificação das pacientes de acordo com os níveis de TSH e com a presença ou não de auto-imunidade tireoidiana, também não foi encontrada diferença na EIM destes subgrupos em relação ao grupo controle.

Nossos achados podem ser explicados pelo fato de que, conforme a maioria dos casos de hipotireoidismo subclínico $(31,32)$, as pacientes do estudo apresentavam hipotireoidismo leve, com média de TSH inferior a $10 \mathrm{mUI} / \mathrm{L}$, além de perfil lipídico semelhante ao das controles. Em apenas 7 pacientes, os níveis de TSH se encontravam acima de $10 \mathrm{mUI} / \mathrm{L}$. Já foi descrito que as alterações lipídicas no hipotireoidismo são mais freqüentes e mais graves nos indivíduos com TSH acima deste valor (33-36).

Além disso, a maioria dos autores propõe que o tratamento do HS deve ser instituído apenas nos indivíduos com TSH acima de 10 a $12 \mathrm{mUI} / \mathrm{L}(32,37$ 39), já que estes obteriam maior benefício com o uso da levotiroxina, no que diz respeito às alterações metabólicas e aos sintomas. Também, a progressão para hipotireoidismo manifesto depende dos níveis de TSH e da presença de auto-imunidade, conforme mostrado por Vanderpump e cols. (4) e por Huber e cols. (38). Neste último trabalho, após um acompanhamento de 9,2 anos, foi observado que 28\% dos pacientes avaliados progrediram para hipotireoidismo manifesto. Destes, nenhum apresentava TSH inferior a $6 \mathrm{mUI} / \mathrm{L}$ e a taxa anual dessa progressão foi de $3,3 \pm$ $1,5 \%$, quando TSH entre 6 e $12 \mathrm{mUI} / \mathrm{L}$, e de $11,4 \pm$ $3,0 \%$, quando $\mathrm{TSH}>12 \mathrm{mUI} / \mathrm{L}$.

A presença de anticorpos em pacientes com HS também já foi associada a um maior risco de lesões ateroscleróticas e à ocorrência de infarto agudo do miocárdio (2). Entretanto, apenas a presença desses anticorpos, sem disfunção tireoidiana, não confere risco elevado para a ocorrência de eventos cardiovasculares $(2,40)$. Em nosso estudo, após a estratificação das pacientes com HS em dois grupos de acordo com a presença ou não de auto-imunidade tireoidiana, não foi observada diferença significativa na medida da EIM. 
Alguns estudos relacionando a EIM carotídea com função tireoidiana já foram realizados. Nagasaki e cols. (41) e Monzani e cols. (42), ao avaliarem a EIM de pacientes com hipotireoidismo manifesto e com hipotireoidismo subclínico, respectivamente, encontraram média da EIM mais elevada do que a observada em nosso estudo.

No primeiro estudo (41), foram avaliados $35 \mathrm{pa}$ cientes com hipotireoidismo manifesto (média de TSH de 74,5 $\pm 10 \mathrm{mUI} / \mathrm{L}$ ) e 35 controles, sem diferença estatística em relação à idade e ao IMC. A medida da EIM nas carótidas comuns dos pacientes foi $0,635 \pm$ $0,018 \mathrm{~mm}$ e a dos controles, $0,559 \pm 0,021 \mathrm{~mm}$, com $\mathrm{p}<0,005$. É importante ressaltar que a média de idade dos pacientes avaliados era de $51,9 \pm 2,2$ anos, enquanto que a das nossas pacientes foi de 43,07 $\pm 9,76$ anos, e que os mesmos apresentavam média de CT e de LDL estatisticamente mais elevadas $(\mathrm{p}=0,04$ para as duas variáveis) do que os controles. Além disso, não foram excluídos indivíduos com história de tabagismo e de HAS. Salonen e col. (43) já demonstraram que a EIM carotídea aumenta conforme a faixa etária e que essa progressão pode ser acelerada na presença de dislipidemia, tabagismo e HAS. Após um ano de tratamento com levotiroxina, houve redução significativa da EIM dos pacientes para $0,552 \pm 0,015 \mathrm{~mm}(\mathrm{p}<0,0001)$, além de melhora do lipidograma.

Monzani e cols. (42) obtiveram resultados semelhantes ao avaliarem 45 indivíduos com hipotireoidismo subclínico e 32 controles pareados por sexo, idade e IMC, sem outros fatores de risco para doença cardiovascular. Os valores encontrados (média entre os valores das carótidas comuns e das bifurcações) foram $0,75 \pm 0,13 \mathrm{~mm}$ nos pacientes e $0,63 \pm$ $0,07 \mathrm{~mm}$, no grupo controle, com $\mathrm{p}<0,001$. A diferença entre as médias de EIM permaneceu significativa mesmo após dividir os pacientes em dois grupos, um com TSH inferior e outro superior a $10 \mathrm{mUI} / \mathrm{L}$. Porém, a diferença entre a EIM dos indivíduos com hipotireoidismo subclínico e os controles deixou de ser significativa quando os primeiros foram estratificados em outros dois grupos, com idade superior ou inferior a 35 anos $(\mathrm{p}=0,20)$. Apesar de a média de idade dos pacientes avaliados ser um pouco menor do que a das nossas pacientes ( $37 \pm 11$ anos vs. 43,07 $\pm 9,76$ anos), os níveis de outros parâmetros sabidamente envolvidos no risco cardiovascular estavam significativamente mais elevados no grupo com hipotireoidismo subclínico (colesterol total, $\mathrm{p}=0,002 ; \mathrm{LDL}, \mathrm{p}=0,0007$; $\mathrm{e}$ apoproteína $\mathrm{B}, \mathrm{p}=0,01)$. Houve melhora da medida da EIM e das alterações metabólicas após seis meses de tratamento com levotiroxina.
Os dados da literatura associando HS e aumento da EIM e os nossos resultados são controversos. Nos estudos em que foi encontrada associação entre hipofunção tireoidiana e aumento da EIM, os pacientes também apresentavam alterações metabólicas que poderiam justificar os resultados encontrados. Nosso estudo não encontrou diferença significativa no perfil lipídico, apoB e Lpa e nos achados da ultra-sonografia carotídea, ao comparar um grupo com HS a indivíduos sem disfunção tireoidiana. A diferença da EIM carotídea permaneceu sem significância estatística mesmo após a estratificação das pacientes de acordo com os níveis de TSH e com a presença ou não de auto-imunidade tireoidiana. Este fato sugere que o HS, quando leve e não associado a alterações metabólicas, mesmo na presença de anticorpos anti-tireoidianos, não gera aumento do risco cardiovascular.

\section{REFERÊNCIAS}

1. Ross DS. Serum thyroid-stimulating hormone measurement for assessment of thyroid function and disease. Endocrinol Metab Clin North Am 2001;30:245-64.

2. Hak AE, Pols HA, Visser TJ, Drexhage HA, Hofman A, Witteman JC. Subclinical hypothyroidism is an independent risk factor for atherosclerosis and myocardial infarction in elderly women: the Rotterdam Study. Ann Intern Med 2000;132:270-8.

3. Parle JV, Franklin JA, Cross KW, Jones SC, Sheppard MC. Prevalence and follow-up of abnormal thyrotropin (TSH) concentrations in the elderly in the United Kingdom. Clin Endocrinol (Oxf) 1991;34:77-83.

4. Vanderpump MPJ, Tunbridge WMG, French JM, Appleton D, Bates D, Clark J. The incidence of thyroid disorders in the community: a twenty-year follow-up of the Whickham Survey. Clin Endocrinol (Oxf) 1995;43:55-68.

5. Sawin CT, Castelli WP, Hershman JM, McNamara $P$, Bacharach $P$. The aging thyroid. Thyroid deficiency in the Framingham Study. Arch Intern Med 1985; 145:1386-8.

6. Althaus BU, Staub JJ, Ryff De Leche A, Oberhansli A, Stahelin HB. LDL/HDL-changes in subclinical hypothyroidism: possible risk factors for coronary heart disease. Clin Endocrinol (Oxf) 1988;28:157-63.

7. Diekman T, Lansberg PJ, Kastelein JJ, Wiersinga WM. Prevalence and correction of hypothyroidism in a large cohort of patients referred for dyslipidemia. Arch Intern Med 1995;155:1490-5.

8. Cabral MD, Costa AJL, Santos M, Vaisman M. Lipid profile in subclinical hypothyroidism. Endocrinologist 2004; 14:121-5.

9. Cappola AR, Ladenson PW. Hypothyroidism and atherosclerosis. J Clin Endocrinol Metab 2003;88(6):2438-44.

10. Expert Panel on detection, evaluation and treatment of high blood cholesterol in adults. Executive summary of the third report of the National Cholesterol Education Program (NCEP) expert panel on detection, evaluation and treatment of high blood cholesterol in adults (adult treatment panel III). JAMA 2001;285(19):2486-97.

11. Lamarche B, Moorjani S, Lupien PJ, Cantin B, Bernard PM, Dagenais GR, et al. Apolipoprotein A-I and B levels and the risk of ischemic heart disease during a five-year follow-up of men in The Québec Cardiovascular Study. Circulation 1996;94:273-8. 
12. Walldius G, Junger I, Holme I, Aastveit AH, Kolar W, Steiner E. High apolipoprotein B, low apolipoprotein A-I, and improvement in the prediction of fatal myocardial infarction (AMORIS study): a prospective study. Lancet 2001;358: 2026-33.

13. Sniderman $A D$, Furberg $C D$, Keech $A$, Roeters van Lennep JE, Frohlich J, Jungner I, et al. Apolipoproteins versus lipids as indices of coronary risk and as targets for statin treatment. Lancet 2003;361:777-80.

14. Armstrong VW, Cremer P, Eberle E, Manke A, Schulze F, Wieland $\mathrm{H}$, et al. The association between serum $\mathrm{Lp}(\mathrm{a})$ concentrations and angiographically assessed coronary atherosclerosis. Atherosclerosis 1986;62:249-57.

15. Bostom AG, Cupples LA, Jenner JL, Ordovas JM, Seman LJ, Wilson PW, et al. Elevated plasma lipoprotein (a) and premature coronary heart disease in Framingham men: a prospective study. JAMA 1996;276:544-8.

16. Ross R. Atherosclerosis - an inflammatoty disease. N Engl J Med 1999;340(2):115-26.

17. Salonen R, Salonen JT. Determinants of carotid intima-media thickness: a population-based ultrasonographic study in Eastern Finnish Men. J Intern Med 1991;229:225-31.

18. O'Leary DH, Polak JF. Intima-media thickness: a tool for athersoclerosis imaging and event prediction. Am J Cardiol 2002;90(suppl):18L-21.

19. Barth JD. An update on carotid ultrasound measurement of intima-media thickness. Am J Cardiol 2002;89(suppl):32B9.

20. Chambless LE, Heiss G, Folsom AR, Rosamond W, Szklo M Sharrett $A R$, et al. Association of coronary heart disease incidence with carotid arterial wall thickness and major risk factors: The Atherosclerosis Risk in Communities (ARIC) Study. Am J Epidemiol 1997; $146: 483-94$.

21. Bots ML, Hoes AW, Koudstaal PJ, Hofman A, Grobbee DE. Common carotid intima-media thickness and risk of stroke and myocardial infarction. The Rotterdam Study. Circulation 1997:96:1432-7.

22. O'Leary DH, Polak JF, Kronmal RA, Manolio TA, Burke GL, Wolfson SK. Carotid-artery intima and media thickness as a risk factor for myocardial infarction and stroke in older adults. N Engl J Med 1999;340(1):14-22

23. Smith SC Jr, Greenland P, Grundy SM. Prevention Conference $\mathrm{V}$ : beyond secondary prevention: identifying the highrisk patient for primary prevention. Circulation 2000:101:111-6.

24. Salonen JT, Salonen R. Ultrasonographycally assessed carotid morphology and the risk of coronary heart disease. Arterioscler Thromb 1991;11:1245-9.

25. Hodis HN, Mack WJ, LaBree L, Selzer RH, Liu CR, Liu CH, et al. The role of carotid arterial intima-media thickness in predicting clinical coronary events. Ann Intern Med 1998; 128:262-9.

26. Gnasso A, Irace C, Mattioli PL, Pujia A. Carotid intima-media thickness and coronary heart disease risk factors. Atherosclerosis 1996;119:7-15.

27. Bots ML, Mulder PGH, Hofman A, van Es, GA, Grobbee DE. Reproducibility of carotid vessel wall thickness measurements. The Rotterdam Study. J Clin Epidemiol 1993;47(8):921-30.

28. Kanters SDJM, Algra A, van Leeuwen MS, Banga JD. Reproducibility of in vivo carotid intima-media thickness measurements. A review. Stroke 1997;28:665-71.

29. Friedwald WT, Levy RI, Friedrickson DS. Estimation of the concentration of low-density lipoprotein cholesterol in plasma without the use of the preparative ultracentrifuge. Clin Chem, 1972;18:499-502.
30. Pignoli P, Tremoli E, Poli A, Oreste P, Paoletti R. Intimal plus medial thickness of the arterial wall: a direct measurement with ultrasound imaging. Circulation 1986;74(6):1399-406.

31. Tunbridge WM, Evered DC, Hall R, Appleton D, Brewis $M$, Clark $\mathrm{F}$, et al. The spectrum of thyroid disease in a community: the Whickham survey. Clin Endocrinol (Oxf) 1977:7:481-93.

32. Chu JW, Crapo LM. The treatment of subclinical hypothyroidism is seldom necessary. J Clin Endocrinol Metab 2001:86(10):4591-9.

33. Oettgen P, Ginsburg GS, Horowitz GL, Pasternak RC. Frequency of hypothyroidism in adults with serum cholesterol levels > $200 \mathrm{mg} / \mathrm{dL}$. Am J Cardiol 1994;73:955-7.

34. Bindels AJGH, Westendorp RGJ, Frölich M, Seidell JC, Blokstra A, Smelt AHM. The prevalence of subclinical hypothyroidism at different total plasma cholesterol levels in middle aged men and women: a need for case finding? Clin Endocrinol 1999:50:217-20.

35. Vierhapper H, Nardi A, Grosser P, Raber W, Gessl A. Lowdensity lipoprotein cholesterol in subclinical hypothyroidism. Thyroid 2000;10:981-4.

36. Duntas LH, Mantzou E, Koutras DA. Circulating levels of oxidized low-density lipoprotein in overt and mild hypothyrooidism. Thyroid 2002;12(11):1003-7.

37. Cooper DS. Subclinical hypothyroidism. N Engl J Med 2001:345(4):260-5.

38. Huber G, Staub JJ, Meier C, Mitrache C, Guglielmetti M, Huber $\mathrm{P}$, et al. Prospective study of the spontaneous course of subclinical hypothyroidism: prognostic value of thyrotropin, thyroid reserve and thyroid antibodies. J Clin Endocrinol Metab 2002;87:3321-6.

39. Surks MI, Ortiz E, Daniels GH, Sawin CT, Col NF, Cobin RH, et al. Subclinical thyroiod disease. Scientific review and guidelines for diagnosis and management. JAMA 2004:291(2):228-38.

40. Wells BJ, Hueston WJ. Are thyroid peroxidase antibodies associated with cardiovascular disease risk in patients with subclinical hypothyroidism? Clin Endocrinol 2005;62:580-

41. Nagasaki $T$, Inaba $M$, Henmi $Y$, Kumeda $Y$, Ueda $M$, Tahara $H$, et al. Decrease in carotid intima-media thickness in hypothyroid patients after normalization of thyroid function. Clin Endocrinol 2003;59:607-12

42. Monzani $F$, Caraccio $N$, Kozàkowà $M$, Dardano $A$, Vittone $F$, Virdis $A$, et al. Effect of levothyroxine replacement on lipid profile and intima-media thickness in subclinical hypothyroidism: a double-blind, placebo-controlled study. J Clin Endocrinol Metab 2004;89(5):2099-106.

43. Salonen R, Salonen JT. Determinants of carotid intima-media thickness: a population-based ultrasonographic study in Eastern Finnish Men. J Intern Med 1991:229:225-31.

Endereço para correspondência:

Carla Amaral de Almeida

Rua Malibu 285, bloco 1, apto. 1204

22793-295 Rio de Janeiro, RJ

E-mail: carlaalm@terra.com.br 